\title{
Influence of alkali and alkaline earth ions on the $O$-alkylation of the lower rim phenolic-OH groups of $p$-tert-butyl-calix[4]arene to result in amide-pendants: Template action of $\mathrm{K}^{+}$and the structure of $\mathrm{K}^{+}$bound tetra-amide derivative crystallized with a $p$-tert-butyl- calix[4]arene anion
}

\author{
AMJAD ALI,${ }^{1,3}$ CHEBROLU P RAO $^{1, *}$ and PHILIPPE GUIONNEAU ${ }^{2}$ \\ ${ }^{1}$ Bioinorganic Laboratory, Department of Chemistry, Indian Institute of Technology Bombay, \\ Powai, Mumbai 400076 \\ ${ }^{2}$ Institut de Chimie de la Matiere Condensee de Bordeaux, UPR 9048 CNRS, Pessac, France \\ ${ }^{3}$ Present address: School of Chemistry and Biochemistry, Thapar University, Patiala 147004 \\ e-mail: cprao@chem.iitb.ac.in
}

MS received 20 December 2007; revised 18 February 2008

\begin{abstract}
Role of alkali and alkaline earth ions on the formation of calix[4]arene-amide derivatives through $O$-alkylation of the lower rim phenolic-OH groups in general and template action of $\mathrm{K}^{+}$in particular have been explored. $\mathrm{Na}^{+}$and $\mathrm{K}^{+}$ions among alkali, and $\mathrm{Ca}^{2+}$ and $\mathrm{Sr}^{2+}$ ions among alkaline earth have shown tetra-amide derivatives bound to metal ion species. Among all these, potassium salts act as template and yields a $\mathrm{K}^{+}$bound tetra-amide derivative where the charge is counter balanced by a calix[4] arene-monoanion and the product is crystallographically characterized. Change in the amide precursor used in these $O$-alkylation reactions has no effect on the type of the amide derivative formed. Also demonstrated is a direct one-step reaction for the preparation of 1,3-di-amide derivative in high yield and low reaction period using $\mathrm{CsHCO}_{3}$.
\end{abstract}

Keywords. $O$-alkylation at the lower rim of calix[4]arene; template action of $\mathrm{K}^{+}$; mono-anion of $p$-tertbutyl-calix[4]arene; alkali and alkaline earth ions.

\section{Introduction}

Metal ion-directed derivatization of calixarenes would be an attractive proposition in building new calixarene based scaffolds since such methodology is expected to reduce the number of steps involved in the synthesis as well as improve the yields when compared to the conventional ones. Synthesis of the lower rim amide derivatives of calix[4]arene is not generally difficult and such derivatives are known and/or expected to complex alkali-, alkaline earth- ${ }^{1-9}$ lanthanide ${ }^{10-13}$ and transition- ${ }^{14-16}$ metal ions as reported in the literature. There are some reports in the literature regarding the synthesis of various types of calix[4]arene-amide derivatives which involve multi-step synthesis. ${ }^{17,18}$ However, there has been no systematic study in the literature that utilizes the catalytic influence of metal ions in the derivatization of calix[4]arene. In this paper, we report for the first time, a systematic investi-

\footnotetext{
*For correspondence
}

gation of the role of alkali and alkaline earth metal ions (as their carbonates, bicarbonates, hydroxides, hydrides) on the different amide derivatives formed via $O$-alkylation at the lower rim of $p$-tert-butylcalix[4]arene. Also this paper deals with the demonstration of the template action of $\mathrm{K}^{+}$in forming tetra-amide derivative and its crystal structure determination that possesses monoanion of p-tertbutyl-calix[4]arene as counter ion.

\section{Experimental}

p-tert-Butyl-calix[4]arene, $\mathbf{1}$ was synthesized as per the reported procedure. ${ }^{19}$ In case, the product is a mixture, individual products were separated either by column chromatography on silica gel or by fractional crystallization or by a combination of both these methods. After the separation, all the individual products were characterized by different techniques including FAB mass and NMR spectra $(400 \mathrm{Mz}$, 
Varian) and the corresponding data is listed in this section.

\subsection{Preliminary crystallographic data for 9}

Crystals of 9 suitable for X-ray determination were obtained by dissolving the product in $\mathrm{CH}_{3} \mathrm{CN}$ and allowing it to evaporate slowly at room temperature. Transparent single crystals of approximate dimensions $0.30 \times 0.10 \times 0.10 \mathrm{~mm}^{3}$ were selected on polarized microscope and mounted on a Bruker-Nonius $\kappa$-CCD diffractometer, Mo-K $\mathrm{K}_{\alpha}$ radiation $(0.71073 \AA)$. The structure was determined by direct methods and the refinement of atomic parameters based on fullmatrix least squares on $F^{2}$ were performed using the SHELX-97 (Sheldrick, G.M., Institüt für Anorganische Chemie der Universität, Tammanstrasse 4, D3400 Göttingen, Germany, 1998) programs. The crystallographic data is deposited at the Cambridge Crystallographic Data Centre with the CCDC code being 280392. Contacts for CCDC are, CCDC, 12 Union Road, CAMBRIDGE CB2 1EZ, UK; deposit@ccdc.cam.ac.uk; http://www.ccdc.cam.ac.uk/ deposit.

\subsection{Synthesis and characterization data for the products}

2.2a 5,11,17,23-Tetra-tert-butyl-25-mono(N,N-diethyl aminocarbonyl)methoxy-26,27,28-trihydroxycalix[4] arene, (2): $0 \cdot 3 \mathrm{~g} \mathrm{NaH}(60 \%$ oil) was taken in 3 neck round bottom flask and was washed several times with dried hexane. Calix[4]arene $(1 \mathrm{~g}, 1.5 \mathrm{mmol})$ was taken in THF/DMF $(40 / 8 \mathrm{~mL})$ in another round bottom flask and stirred at $60^{\circ} \mathrm{C}$ for $1 \mathrm{~h}$ and then it was allowed to cool down to RT and the contents were transferred to the flask containing $\mathrm{NaH}$ and $\alpha-C l$ DMA $(0.60 \mathrm{~g}, 4.5 \mathrm{mmol})$ and refluxed for $3 \mathrm{~h}$ and the reaction mixture was allowed to cool to RT and product formed was precipitated out by adding water into the reaction mixture. Crude product was isolated by filtration and separated by column chromatography on silica gel using ethylacetate/pet ether solvent system $(10 / 90)$ to yield 2, 3 and 4. 2: Yield 46\%. m.p. $120-122^{\circ} \mathrm{C} . \quad \mathrm{C}_{50} \mathrm{H}_{67} \mathrm{NO}_{5} \cdot 2 \mathrm{CH}_{3} \mathrm{COO} \quad \mathrm{C}_{2} \mathrm{H}_{5} \quad(938 \cdot 24)$ : calc.: C 74.24, H 8.92, N 1.49; found: C 74.32, H 8.73, N 1.39\%. FTIR: $\left(\mathrm{KBr}, \mathrm{cm}^{-1}\right): 1653\left(v_{\mathrm{C}=\mathrm{O}}\right), 3343$ $\left(v_{\mathrm{OH}}\right) .{ }^{1} \mathrm{H}$ NMR: $\left(\mathrm{CDCl}_{3}, \delta \mathrm{ppm}\right): 7.08(s, 2 \mathrm{H}, \mathrm{Ar}-H)$, $7.04(s, 2 \mathrm{H}, \operatorname{Ar}-H), 6.95(d, J=3.33 \mathrm{~Hz}, 2 \mathrm{H}, \operatorname{Ar}-H)$, $4.96\left(s, 2 \mathrm{H}, \mathrm{OCH}_{2} \mathrm{CO}\right), 4.59(d, J=12.82 \mathrm{~Hz}, 2 \mathrm{H}$, $\left.\mathrm{Ar}-\mathrm{CH}_{2}-\mathrm{Ar}\right), 4.34\left(d, J=13.55 \mathrm{~Hz}, 2 \mathrm{H}, \mathrm{Ar}-\mathrm{CH}_{2}-\mathrm{Ar}\right)$, $3.54\left(q, J=7.33,6.96,6.96 \mathrm{~Hz}, 2 \mathrm{H}, \mathrm{NCH}_{2}\right), 3.39(d$, $\left.J=13.55 \mathrm{~Hz}, 2 \mathrm{H}, \mathrm{Ar}-\mathrm{CH}_{2}-\mathrm{Ar}\right), 3.37(d, J=12.82 \mathrm{~Hz}$, $\left.2 \mathrm{H}, \operatorname{Ar}-\mathrm{CH}_{2}-\mathrm{Ar}\right), 3.27(q, J=6.96,7.33,6.96 \mathrm{~Hz}$, $\left.2 \mathrm{H}, \mathrm{NCH}_{2}\right), 1 \cdot 18\left(m, 42 \mathrm{H}, \mathrm{C}\left(\mathrm{CH}_{3}\right)_{3}\right.$ and $\left.\mathrm{NCH}_{2} \mathrm{CH}_{3}\right)$. ${ }^{13} \mathrm{C}$ NMR: $\left(\mathrm{CDCl}_{3}, \delta \mathrm{ppm}\right): 13.05,14 \cdot 15\left(\mathrm{NCH}_{2} \mathrm{CH}_{3}\right)$, $29.75\left(\mathrm{Ar}-\mathrm{CH}_{2}-\mathrm{Ar}\right), 31.32,31.50,31.60\left(\mathrm{C}\left(\mathrm{CH}_{3}\right)_{3}\right)$, $32 \cdot 91,33 \cdot 14,34 \cdot 20,33 \cdot 95\left(\mathrm{C}_{\left.\left(\mathrm{CH}_{3}\right)_{3}\right), 40 \cdot 41,40 \cdot 66}\right.$ $\left(\mathrm{NCH}_{2}\right), 72.23\left(\mathrm{OCH}_{2}\right), 125.61,125.86,126.31$ $\left(\mathrm{C}_{m}\right), 127.93(d), 128.33,134.02 \quad\left(\mathrm{C}_{o}\right), 142.84$, $143.05,147.79\left(\mathrm{C}_{p}\right), 148.27,148.66,151.99\left(\mathrm{C}_{i}\right)$, $168.44(\mathrm{C}=\mathrm{O})$. FAB-MS: $m / z$ (intensity $(\%)$, fragment) $761\left(70,\left[\mathrm{M}-\mathrm{H}^{+}\right]^{+}\right)$.

\section{2b 5,11,17,23-Tetra-tert-butyl-25,27-bis(N,N-diethyl} aminocarbonyl)methoxy-26,28-dihydroxycalix [4] arene, (3): Yield 5\%. m.p. $>240^{\circ} \mathrm{C}$ (decomp). $\mathrm{C}_{56} \mathrm{H}_{78} \mathrm{~N}_{2} \mathrm{O}_{6}$ (875.23): calc.: C 76.85, H 8.98, N 3.20; found: C 76.60, H 8.79, N 3.35\%. FTIR: $\left(\mathrm{KBr}, \mathrm{cm}^{-1}\right)$ : $1661\left(v_{\mathrm{C}=\mathrm{O}}\right), 3366\left(v_{\mathrm{OH}}\right) .{ }^{1} \mathrm{H}$ NMR: $\left(\mathrm{CDCl}_{3}, \delta \mathrm{ppm}\right)$ : $6.89(s, 4 \mathrm{H}, \mathrm{Ar}-\mathrm{H}), 6.86(s, 4 \mathrm{H}, \mathrm{Ar}-\mathrm{H}), 4.82(s, 4 \mathrm{H}$, $\left.\mathrm{OCH}_{2} \mathrm{CO}\right), 4.45\left(d, J=12.82 \mathrm{~Hz}, 2 \mathrm{H}, \mathrm{Ar}-\mathrm{CH}_{2}-\mathrm{Ar}\right)$, $3.45\left(q, J=6.96,6.96,6.59 \mathrm{~Hz}, 8 \mathrm{H}, \mathrm{NCH}_{2}\right), 3.27(d$, $J=13.19 \mathrm{~Hz}, \quad 4 \mathrm{H}, \quad$ Ar- $\left.\mathrm{CH}_{2}-\mathrm{Ar}\right), 1.18(m, 30 \mathrm{H}$, $\mathrm{C}\left(\mathrm{CH}_{3}\right)_{3}$ and $\left.\mathrm{NCH}_{2} \mathrm{CH}_{3}\right), 1.036\left(s, 18 \mathrm{H}, \mathrm{C}\left(\mathrm{CH}_{3}\right)_{3}\right)$. ${ }^{13} \mathrm{C}$ NMR: $\left(\mathrm{CDCl}_{3}, \delta \mathrm{ppm}\right): 13.04,14.36\left(\mathrm{NCH}_{2} \mathrm{CH}_{3}\right)$,

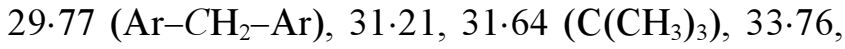
$33.99 \quad\left(\mathrm{C}\left(\mathrm{CH}_{3}\right)_{3}\right), \quad 40.32, \quad 41.30 \quad\left(\mathrm{NCH}_{2}\right), \quad 73.58$ $\left(\mathrm{OCH}_{2}\right), 124.93,125.68\left(\mathrm{C}_{m}\right), 127.82,133.28\left(\mathrm{C}_{o}\right)$, $141.23,146.84\left(\mathrm{C}_{p}\right), 150.12,151.99\left(\mathrm{C}_{i}\right), 167.96$ $(\mathrm{C}=\mathrm{O})$. FAB-MS: $m / z$ (intensity $(\%)$, fragment) 875 $\left(100,[\mathrm{M}]^{+}\right)$.

\section{2c 5,11,17,23-Tetra-tert-butyl-25,26-bis $(N, N$-di-} ethylaminocarbonyl)methoxy-27,28-dihydroxycalix [4] arene, (4): Yield 36\%. m.p. $213-216^{\circ} \mathrm{C}$. $\mathrm{C}_{56} \mathrm{H}_{78} \mathrm{~N}_{2} \mathrm{O}_{6} \cdot \mathrm{H}_{2} \mathrm{O}(893.23)$ : C 75.03, H 9.40, N 4.02; found: C 75.30, H 9.22, N 3.85\%. FTIR: $\left(\mathrm{KBr}, \mathrm{cm}^{-1}\right)$ : $1645\left(v_{\mathrm{C}=\mathrm{O}}\right), 3343\left(v_{\mathrm{OH}}\right) .{ }^{1} \mathrm{H}$ NMR: $\left(\mathrm{CDCl}_{3}, \delta \mathrm{ppm}\right)$ : $6.91(d, J=2.19 \mathrm{~Hz}, 2 \mathrm{H}, \operatorname{Ar}-\mathrm{H}), 6.86(d, J=2.19$ $\mathrm{Hz}, 2 \mathrm{H}, \mathrm{Ar}-\mathrm{H}), 6.78(d d, J=2.56,2.19 \mathrm{~Hz}, 4 \mathrm{H}, \mathrm{Ar}-$ $\mathrm{H}), 5.27\left(d, J=14.65 \mathrm{~Hz}, 2 \mathrm{H}, \mathrm{OCH}_{2} \mathrm{CO}\right), 4.88(d$, $\left.J=13.91 \mathrm{~Hz}, \quad 1 \mathrm{H}, \quad \mathrm{Ar}-\mathrm{CH}_{2}-\mathrm{Ar}\right), \quad 4.67 \quad(d, \quad J=$ $\left.14.65 \mathrm{~Hz}, 2 \mathrm{H}, \mathrm{OCH}_{2} \mathrm{CO}\right), 4.65(d, J=12.81 \mathrm{~Hz}, 2 \mathrm{H}$, Ar- $\left.\mathrm{CH}_{2}-\mathrm{Ar}\right), 4.32\left(d, J=13.55 \mathrm{~Hz}, 1 \mathrm{H}, \mathrm{Ar}-\mathrm{CH}_{2}-\mathrm{Ar}\right)$, $3.35\left(m, 8 \mathrm{H}, \mathrm{NCH}_{2}\right), 3.23(d d, J=13.92,12.82 \mathrm{~Hz}$, $\left.4 \mathrm{H}, \quad \mathrm{Ar}-\mathrm{CH}_{2}-\mathrm{Ar}\right), \quad 1.20 \quad\left(m, 36 \mathrm{H}, \quad \mathrm{C}\left(\mathrm{CH}_{3}\right)_{3}\right.$ and $\left.\mathrm{NCH}_{2} \mathrm{CH}_{3}\right), 1.01\left(s, 18 \mathrm{H}, \mathrm{C}\left(\mathrm{CH}_{3}\right)_{3}\right) .{ }^{13} \mathrm{C} \mathrm{NMR}:\left(\mathrm{CDCl}_{3}\right.$, $\delta \mathrm{ppm}): 13.08,14.21\left(\mathrm{NCH}_{2} \mathrm{CH}_{3}\right), 29.74\left(\mathrm{Ar}-\mathrm{CH}_{2}-\right.$ Ar), 31.29, $31.59\left(\mathrm{C}_{\left.\left(\mathrm{CH}_{3}\right)_{3}\right), 32.01(d), 33.73,33.89}\right.$ $\left(\mathrm{C}\left(\mathrm{CH}_{3}\right)_{3}\right), 40 \cdot 27,40.91 \quad\left(\mathrm{NCH}_{2}\right), 73.34\left(\mathrm{OCH}_{2}\right)$, $124 \cdot 88,125 \cdot 03,125 \cdot 51\left(\mathrm{C}_{m}\right), 126 \cdot 01,127 \cdot 24,127 \cdot 57$ 
$\left(\mathrm{C}_{o}\right), 132.89,134.04,141.21\left(\mathrm{C}_{p}\right), 145.91,149.06$, $154.25\left(\mathrm{C}_{i}\right), 169 \cdot 36(\mathrm{C}=\mathrm{O})$. FAB-MS: $m / z$ (intensity (\%), fragment) $875\left(70,[\mathrm{M}]^{+}\right)$.

2.2d 5,11,17,23-Tetra-tert-butyl-25,26,27,28- tetrakis(N,N-diethylaminocarbonyl) methoxycalix [4]arene, (5): Yield $90 \%$. m.p. $170-175^{\circ} \mathrm{C}$. FTIR: $\left(\mathrm{KBr}, \mathrm{cm}^{-1}\right)$ : $1661\left(v_{\mathrm{C}=\mathrm{O}}\right), 3440\left(v_{\mathrm{OH}}\right) .{ }^{1} \mathrm{H}$ NMR: $\left(\mathrm{CDCl}_{3}, \delta \mathrm{ppm}\right)$ : $6.81(s, 8 \mathrm{H}, \mathrm{Ar}-\mathrm{H}), 5.20(d, J=12 \cdot 2 \mathrm{~Hz}, 4 \mathrm{H}, \mathrm{Ar}-$ $\left.\mathrm{CH}_{2}-\mathrm{Ar}\right), 5.02\left(s, 8 \mathrm{H}, \mathrm{OCH}_{2} \mathrm{CO}\right), 3.35(m, 16 \mathrm{H}$, $\left.\mathrm{NCH}_{2}\right), 3.20\left(d, J=12.21 \mathrm{~Hz}, 4 \mathrm{H}, \mathrm{Ar}-\mathrm{CH}_{2}-\mathrm{Ar}\right)$, $1.14\left(m, 60 \mathrm{H}, \mathrm{C}\left(\mathrm{CH}_{3}\right)_{3}\right.$ and $\left.\mathrm{NCH}_{2} \mathrm{CH}_{3}\right)$. FAB-MS: $\mathrm{m} / z$ (intensity $(\%)$, fragment) $1102\left(100,[\mathrm{M}]^{+}\right)$.

2.2e $\quad[(5,11,17,23$-Tetra-tert-butyl-25,26,27,28-tetrakis ( $N, N$-diethylaminocarbonyl) methoxycalix [4]arene) sodium(I)]hydroxide, (6): A mixture of 1 and $\mathrm{NaOH}$ or $\mathrm{NaHCO}_{3}$ (1:5) was stirred at RT in dry $\mathrm{CH}_{3} \mathrm{CN}$. To this, $\alpha$-Cl-DMA (5 eq to 1 ) was added and reaction mixture was refluxed for $36 \mathrm{~h}$. The reaction mixture was then allowed to cool down to RT, filtered through celite and the filtrate was evaporated under vacuum to result in a solid product. Yield $85 \%$. m.p. $>240^{\circ} \mathrm{C}$ (decomp.). $\mathrm{C}_{68} \mathrm{H}_{100} \mathrm{~N}_{4} \mathrm{O}_{8} \mathrm{NaOH}$. $\mathrm{H}_{2} \mathrm{O}(1159.66)$ : C 70.43, H 8.95, N 4.83, Na 1.98; found: $\mathrm{C} 70.30, \mathrm{H} 9.02, \mathrm{~N} 4.85, \mathrm{Na} 1.95 \%$. FTIR: $\left(\mathrm{KBr}, \mathrm{cm}^{-1}\right): 1656\left(v_{\mathrm{C}=\mathrm{O}}\right), 3430\left(v_{\mathrm{OH}}\right) .{ }^{1} \mathrm{H}$ NMR: $\left(\mathrm{CDCl}_{3}, \delta \mathrm{ppm}\right): 7 \cdot 15(s, 8 \mathrm{H}, \mathrm{Ar}-\mathrm{H}), 4.52(s, 8 \mathrm{H}$, $\left.\mathrm{OCH}_{2} \mathrm{CO}\right), 4.43\left(d, J=12.21 \mathrm{~Hz}, 4 \mathrm{H}, \mathrm{Ar}-\mathrm{CH}_{2}-\mathrm{Ar}\right)$, $3.42\left(q, J=6.10,7.33,7.33,8 \mathrm{H}, \mathrm{NCH}_{2}\right), 3.32(d$, $\left.J=12.21 \mathrm{~Hz}, 4 \mathrm{H}, \mathrm{Ar}-\mathrm{CH}_{2}-\mathrm{Ar}\right), 3 \cdot 17(q, J=6.09$, $\left.7.33,7.43,8 \mathrm{H}, \mathrm{NCH}_{2}\right), 1 \cdot 14\left(m, 60 \mathrm{H}, \mathrm{C}\left(\mathrm{CH}_{3}\right)_{3}\right.$ and $\left.\mathrm{NCH}_{2} \mathrm{CH}_{3}\right) .{ }^{13} \mathrm{C}$ NMR: $\left(\mathrm{CDCl}_{3}, \delta \mathrm{ppm}\right): 12 \cdot 80,14.04$ $\left(\mathrm{NCH}_{2} \mathrm{CH}_{3}\right), 30.02\left(\mathrm{Ar}-\mathrm{CH}_{2}-\mathrm{Ar}\right), 31.17\left(\mathrm{C}\left(\mathrm{CH}_{3}\right)_{3}\right)$, $34.19 \quad\left(\mathrm{C}_{\left.\left(\mathrm{CH}_{3}\right)_{3}\right),}, 40 \cdot 13, \quad 40.54 \quad\left(\mathrm{NCH}_{2}\right), \quad 73.98\right.$ $\left(\mathrm{OCH}_{2}\right), 125.64\left(\mathrm{C}_{m}\right), 134.82\left(C_{\mathrm{o}}\right), 134.34\left(\mathrm{C}_{p}\right)$, $148.13\left(\mathrm{C}_{i}\right), 167.95(\mathrm{C}=\mathrm{O})$. FAB-MS: $m / z$ (intensity (\%), fragment) $1125\left(100,[\mathrm{M}]^{+}\right)$.

$2.2 \mathrm{f} \quad[(5,11,17,23$-tetra-tert-butyl-25,26,27,28-tetrakis(N,N-diethylaminocarbonyl) methoxycalix[4]arene) potassium(I)]hydroxide, (7): This was synthesized by the same procedure as described for $\mathbf{6}$, but using $\mathrm{KHCO}_{3}$ in place of $\mathrm{NaHCO}_{3}$. Yield 85\%. m.p. 190$195^{\circ} \mathrm{C} . \mathrm{C}_{68} \mathrm{H}_{100} \mathrm{~N}_{4} \mathrm{O}_{8} \mathrm{KOH} \cdot \mathrm{H}_{2} \mathrm{O}$ (1174.66): C 69.47, $\mathrm{H} 8.83$, N 4.77, K 3.33; found: C 69.30, H 8.92, N 4.88, K 3.55\%. FTIR: $\left(\mathrm{KBr}, \mathrm{cm}^{-1}\right): 1654\left(v_{\mathrm{C}=0}\right)$, $3430\left(v_{\mathrm{OH}}\right) .{ }^{1} \mathrm{H}$ NMR: $\left(\mathrm{CDCl}_{3}, \delta \mathrm{ppm}\right): 7 \cdot 13(s, 8 \mathrm{H}$, $\mathrm{Ar}-\mathrm{H}), 4.69\left(\mathrm{~m}, 12 \mathrm{H}, \mathrm{OCH}_{2} \mathrm{CO}\right.$ and $\left.\mathrm{Ar}-\mathrm{CH}_{2}-\mathrm{Ar}\right)$, $3.44\left(q, J=5 \cdot 19,5 \cdot 19,6 \cdot 72,8 \mathrm{H}, \mathrm{NCH}_{2}\right), 3.32(d$, $\left.J=12.22 \mathrm{~Hz}, 4 \mathrm{H}, \mathrm{Ar}-\mathrm{CH}_{2}-\mathrm{Ar}\right), 3 \cdot 18(q, J=5 \cdot 19$, $\left.5 \cdot 32,6 \cdot 82,8 \mathrm{H}, \mathrm{NCH}_{2}\right), 1 \cdot 19\left(m, 60 \mathrm{H}, \mathrm{C}\left(\mathrm{CH}_{3}\right)_{3}\right.$ and $\left.\mathrm{NCH}_{2} \mathrm{CH}_{3}\right) .{ }^{13} \mathrm{C}$ NMR: $\left(\mathrm{CDCl}_{3}, \delta \mathrm{ppm}\right): 12 \cdot 82,14.08$ $\left(\mathrm{NCH}_{2} \mathrm{CH}_{3}\right), 29.27\left(\mathrm{Ar}-\mathrm{CH}_{2}-\mathrm{Ar}\right), 31.47\left(\mathrm{C}\left(\mathrm{CH}_{3}\right)_{3}\right)$, $34.02 \quad\left(\mathrm{C}_{\left.\left(\mathrm{CH}_{3}\right)_{3}\right),} \quad 40.42, \quad 40.98 \quad\left(\mathrm{NCH}_{2}\right), \quad 73.39\right.$ $\left(\mathrm{OCH}_{2}\right), 125.65\left(\mathrm{C}_{m}\right), 134.45\left(\mathrm{C}_{o}\right), 134.34\left(\mathrm{C}_{p}\right)$, 147.28 $\left(\mathrm{C}_{i}\right), 167.77(\mathrm{C}=\mathrm{O})$. FAB-MS: $m / z$ (intensity (\%), fragment) $1141\left(100,[\mathrm{M}]^{+}\right)$.

\section{2g $\quad[(5,11,17,23-$ Tetra-tert-butyl-25,26,27,28-} tetrakis ( $N, N$-diethylaminocarbonyl) methoxy-calix [4] arene)sodium(I)] 5,11,17,23-tetra-tert-butyl-25, 26, 27, 28-tetra hydroxycalix[4]arene-monoanion, (8): This was synthesized by the same procedure as described for 6, but using $\mathrm{Na}_{2} \mathrm{CO}_{3}$ in place of $\mathrm{NaHCO}_{3}$. Resultant product was isolated and purified by fractional crystallization to yield $\mathbf{5}$ and $\mathbf{8}$. The characterization details for $\mathbf{5}$ are given earlier in this experimental part. The details for $\mathbf{8}$ are: Yield 35\%. m.p. $>175^{\circ} \mathrm{C}$ (decomp.). $\mathrm{C}_{112} \mathrm{H}_{156} \mathrm{~N}_{4} \mathrm{O}_{12} \mathrm{Na} .5 \mathrm{CH}_{3} \mathrm{CN}$ (1978.71): C 74.05, H 8.71, N 6.37; Found: C 74.16, H 9.01, N 6.42\%. FTIR: $\left(\mathrm{KBr}, \mathrm{cm}^{-1}\right): 1658\left(v_{\mathrm{C}=\mathrm{O}}\right)$, $3429\left(v_{\mathrm{OH}}\right) .{ }^{1} \mathrm{H}$ NMR: $\left(\mathrm{CDCl}_{3}, \delta \mathrm{ppm}\right): 7 \cdot 18(s, 8 \mathrm{H}$, $\mathrm{Ar}-\mathrm{H}), 6.95(s, 8 \mathrm{H}, \mathrm{Ar}-\mathrm{H}), 4.99\left(m, 16 \mathrm{H}, \mathrm{OCH}_{2} \mathrm{CO}\right.$ and $\left.\mathrm{Ar}-\mathrm{CH}_{2}-\mathrm{Ar}\right), 4.42(d, J=12.21 \mathrm{~Hz}, 4 \mathrm{H}, \mathrm{Ar}-$ $\left.\mathrm{CH}_{2}-\mathrm{Ar}\right), 3.42(q, J=7.33,6.10,7.33 \mathrm{~Hz}, 8 \mathrm{H}$, $\left.\mathrm{NCH}_{2}\right), 3.31\left(d, J=12.21 \mathrm{~Hz}, 4 \mathrm{H}, \mathrm{Ar}-\mathrm{CH}_{2}-\mathrm{Ar}\right)$, $3.22\left(d, J=12.21 \mathrm{~Hz}, 4 \mathrm{H}, \mathrm{Ar}-\mathrm{CH}_{2}-\mathrm{Ar}\right), 3.16(q$, $\left.J=6.11,7.32,7.33 \mathrm{~Hz}, 8 \mathrm{H}, \mathrm{NCH}_{2}\right), 1.19(m, 96 \mathrm{H}$, $\mathrm{C}\left(\mathrm{CH}_{3}\right)_{3}$ and $\left.\mathrm{NCH}_{2} \mathrm{CH}_{3}\right) .{ }^{13} \mathrm{C} \mathrm{NMR}$ : $\left(\mathrm{CDCl}_{3}, \delta \mathrm{ppm}\right)$ : 13.07, $14.17 \quad\left(\mathrm{NCH}_{2} \mathrm{CH}_{3}\right), \quad 30.13 \quad\left(\mathrm{Ar}-\mathrm{CH}_{2}-\mathrm{Ar}\right)$, $31.29,31.39\left(\mathrm{C}\left(\mathrm{CH}_{3}\right)_{3}\right), 32.58,34.01 \quad\left(\mathrm{C}\left(\mathrm{CH}_{3}\right)_{3}\right)$, $40 \cdot 23,40.62\left(\mathrm{NCH}_{2}\right), 74.11\left(\mathrm{OCH}_{2}\right), 125 \cdot 71,125.93$ $\left(\mathrm{C}_{m}\right), 127.69,127.97 \quad\left(\mathrm{C}_{o}\right), 134.94,146.64\left(\mathrm{C}_{p}\right)$, $158.35,151.30\left(\mathrm{C}_{i}\right), 167.95(\mathrm{C}=\mathrm{O})$. FAB-MS: $m / z$ (intensity (\%), fragment) 1124 (100, [M-Calix[4] arenemonoanion $]^{+}$).

$2.2 \mathrm{~h} \quad[(5,11,17,23$-Tetra-tert-butyl-25,26,27,28-tetrakis (N,N-diethylaminocarbonyl) methoxycalix[4]arene) potassium(I)] 5,11,17,23-tetra-tert-butyl-25, 26, 27, 28-tetrahydroxycalix [4]arene-monoanion, (9): This was synthesized by the same procedure as described for 6, but using $\mathrm{K}_{2} \mathrm{CO}_{3}$ in place of $\mathrm{NaHCO}_{3}$. Yield 77\%. m.p. $>185^{\circ} \mathrm{C}$ (decomp.). $\quad \mathrm{C}_{112} \mathrm{H}_{156} \mathrm{~N}_{4} \mathrm{O}_{12} \mathrm{~K}$. $5 \mathrm{CH}_{3} \mathrm{CN}$ (1994.82): C 73.46, H 8.64, N 6.32; found: C 73.16, H 9.01, N 6.52\%. FTIR: $\left(\mathrm{KBr}, \mathrm{cm}^{-1}\right): 1650$ $\left(v_{\mathrm{C}=\mathrm{O}}\right), 3429\left(v_{\mathrm{OH}}\right) .{ }^{1} \mathrm{H}$ NMR: $\left(\mathrm{CDCl}_{3}, \delta \mathrm{ppm}\right): 13 \cdot 17$ (br, 3H, Ar- $\mathrm{OH}, \mathrm{D}_{2} \mathrm{O}$ exchanged), $7 \cdot 15(s, 8 \mathrm{H}, \mathrm{Ar}-$ $\mathrm{H}), 6.92(\mathrm{~s}, 8 \mathrm{H}, \mathrm{Ar}-\mathrm{H}), 4.57\left(\mathrm{~m}, 16 \mathrm{H}, \mathrm{OCH}_{2} \mathrm{CO}\right.$ and $\left.\mathrm{Ar}-\mathrm{CH}_{2}-\mathrm{Ar}\right), 3.43(q, J=6.96,7.33,6.96 \mathrm{~Hz}, 8 \mathrm{H}$, $\left.\mathrm{NCH}_{2}\right), 3.325\left(d, J=12.45 \mathrm{~Hz}, 4 \mathrm{H}, \mathrm{Ar}-\mathrm{CH}_{2}-\mathrm{Ar}\right)$, 
$3.18\left(m, 12 \mathrm{H}, \mathrm{NCH}_{2}\right.$ and $\left.\mathrm{Ar}-\mathrm{CH}_{2}-\mathrm{Ar}\right), 1.20(\mathrm{~m}$, $96 \mathrm{H}, \mathrm{C}\left(\mathrm{CH}_{3}\right)_{3}$ and $\left.\mathrm{NCH}_{2} \mathrm{CH}_{3}\right) .{ }^{13} \mathrm{C}$ NMR: $\left(\mathrm{CDCl}_{3}, \delta\right.$ ppm): 13.08, $14.21 \quad\left(\mathrm{NCH}_{2} \mathrm{CH}_{3}\right), 29.41\left(\mathrm{Ar}-\mathrm{CH}_{2}-\right.$

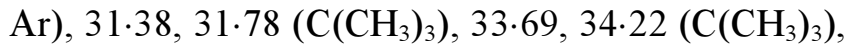
$40 \cdot 70,41 \cdot 15\left(\mathrm{NCH}_{2}\right), 73.54\left(\mathrm{OCH}_{2}\right), 124.59,125 \cdot 78$ $\left(\mathrm{C}_{m}\right), 130.35,134.61\left(\mathrm{C}_{o}\right), 139 \cdot 24,147.34\left(\mathrm{C}_{p}\right)$, $151.31,153.07\left(\mathrm{C}_{i}\right), 167.95(\mathrm{C}=\mathrm{O})$. FAB-MS: $\mathrm{m} / \mathrm{z}$ (intensity (\%), fragment) $1786\left(3,[\mathrm{M}]^{+}\right), 1141(100$, [M-calix[4]arene-monoanion] ${ }^{+}$).

$2.2 \mathrm{i} \quad[(5,11,17,23$-Tetra-tert-butyl-25, 26, 27,28-tetrakis (N,N-diethylaminocarbonyl) methoxycalix[4] arene)calcium(II)] hydroxide, (10): This was synthesized by the same procedure as described for $\mathbf{6}$, but using $\mathrm{Ca}(\mathrm{OH})_{2}$ in place of $\mathrm{NaHCO}_{3}$. Yield $80 \%$. m.p. $>200^{\circ} \mathrm{C}$ (decomp.). $\mathrm{C}_{68} \mathrm{H}_{100} \mathrm{~N}_{4} \mathrm{O}_{8} \mathrm{Ca}(\mathrm{OH})_{2}$. $\mathrm{CH}_{3} \mathrm{CN}$ (1215.74): C 69.10, H 8.70, N 5.76, Ca 3.29; found: C 69.30, H 8.65, N 5.98, Ca 3.45\%. FTIR: $\left(\mathrm{KBr} \mathrm{cm}{ }^{-1}\right): 1632\left(v_{\mathrm{C}=\mathrm{O}}\right), 3420\left(v_{\mathrm{OH}}\right) .{ }^{1} \mathrm{H}$ NMR: $\left(\mathrm{CDCl}_{3}, \delta \mathrm{ppm}\right): 7.17(s, 8 \mathrm{H}, \mathrm{Ar}-\mathrm{H}), 4.96(s$, $\left.8 \mathrm{H}, \mathrm{OCH}_{2} \mathrm{CO}\right), 4.29\left(d, J=13.55 \mathrm{~Hz}, 4 \mathrm{H}, \mathrm{Ar}-\mathrm{CH}_{2}-\right.$ Ar), $3.41\left(m, J=5 \cdot 19,5 \cdot 19,6 \cdot 72,20 \mathrm{H}, \mathrm{NCH}_{2}\right.$ and $\left.\mathrm{Ar}-\mathrm{CH}_{2}-\mathrm{Ar}\right) 1.16\left(\mathrm{~m}, 60 \mathrm{H}, \mathrm{C}\left(\mathrm{CH}_{3}\right)_{3}\right.$ and $\left.\mathrm{NCH}_{2} \mathrm{CH}_{3}\right)$. ${ }^{13} \mathrm{C}$ NMR: $\left(\mathrm{CDCl}_{3}, \delta \mathrm{ppm}\right): 12.77,13.83\left(\mathrm{NCH}_{2} \mathrm{CH}_{3}\right)$, $31.08\left(\mathrm{Ar}-\mathrm{CH}_{2}-\mathrm{Ar}\right), 31.29\left(\mathrm{C}\left(\mathrm{CH}_{3}\right)_{3}\right), 34.23\left(\mathrm{C}_{\left.\left(\mathrm{CH}_{3}\right)_{3}\right)}\right)$, 41.46, $42.07\left(\mathrm{NCH}_{2}\right), 76.84\left(\mathrm{OCH}_{2}\right), 126.54\left(\mathrm{C}_{m}\right)$, $133.51\left(\mathrm{C}_{o}\right), 134.18\left(\mathrm{C}_{p}\right), 148.90\left(\mathrm{C}_{i}\right), 169 \cdot 19(\mathrm{C}=\mathrm{O})$. FAB-MS: $m / z$ (intensity (\%), fragment) $1140(25$, $\left.[\mathrm{M}-\mathrm{H}]^{+}\right)$.

$2.2 \mathrm{j} \quad[(5,11,17,23$-Tetra-tert-butyl-25,26,27,28-tetrakis (N,N-diethylaminocarbonyl) methoxycalix[4] arene)strontium(II)]hydroxide, (11): This was synthesized by the same procedure as described for $\mathbf{6}$, but using $\mathrm{Sr}(\mathrm{OH})_{2}$ in place of $\mathrm{NaHCO}_{3}$. Yield $80 \%$. m.p. $>175^{\circ} \mathrm{C}$ (decomp). $\quad \mathrm{C}_{68} \mathrm{H}_{100} \mathrm{~N}_{4} \mathrm{O}_{8} \mathrm{Sr}(\mathrm{OH})_{2} \cdot \mathrm{CH}_{3} \mathrm{CN}$ (1263.69): C 65.50, H 8.37, N 5.54, Sr 6.93; found: C 65.30, H 8.55, N 5.88, Sr 6.75\%. FTIR: $\left(\mathrm{KBr}, \mathrm{cm}^{-1}\right)$ : $1635\left(v_{\mathrm{C}=\mathrm{O}}\right), 3420\left(v_{\mathrm{OH}}\right) .{ }^{1} \mathrm{H}$ NMR: $\left(\mathrm{CDCl}_{3}, \delta \mathrm{ppm}\right)$ : $7.21(s, 8 \mathrm{H}, \mathrm{Ar}-\mathrm{H}), 4.93\left(s, 8 \mathrm{H}, \mathrm{OCH}_{2} \mathrm{CO}\right), 4.32(d$, $\left.J=12.79 \mathrm{~Hz}, 4 \mathrm{H}, \mathrm{Ar}-\mathrm{CH}_{2}-\mathrm{Ar}\right), 3.44(m, J=5.19$, $5 \cdot 19,6 \cdot 72,20 \mathrm{H}, \mathrm{NCH}_{2}$ and $\left.\mathrm{Ar}-\mathrm{CH}_{2}-\mathrm{Ar}\right) 1.16(\mathrm{~m}$, $60 \mathrm{H}, \mathrm{C}\left(\mathrm{CH}_{3}\right)_{3}$ and $\left.\mathrm{NCH}_{2} \mathrm{CH}_{3}\right) \cdot{ }^{13} \mathrm{C}$ NMR: $\left(\mathrm{CDCl}_{3}, \delta\right.$ ppm): $12.89,13.87\left(\mathrm{NCH}_{2} \mathrm{CH}_{3}\right), 31.16\left(\mathrm{Ar}-\mathrm{CH}_{2}-\right.$ Ar), $31.43\left(\mathrm{C}\left(\mathrm{CH}_{3}\right)_{3}\right), 34.32\left(\mathrm{C}_{\left.\left(\mathrm{CH}_{3}\right)_{3}\right), 41.46,42.05}\right.$ $\left(\mathrm{NCH}_{2}\right), 76.85\left(\mathrm{OCH}_{2}\right), 126.42\left(\mathrm{C}_{m}\right), 127.70\left(\mathrm{C}_{o}\right)$, $134.52\left(\mathrm{C}_{p}\right), 148.99\left(\mathrm{C}_{i}\right), 169.19(\mathrm{C}=\mathrm{O})$. FAB-MS: $m / z$ (intensity (\%), fragment) $1188\left(25,[\mathrm{M}]^{+}\right)$.

$2.2 \mathrm{k} \quad[(5,11,17,23-$ Tetra-tert-butyl-25,26,27,28-tetrakis (N,N-dimethylaminocarbonyl) methoxycalix [4]
arene)sodium(I)]Hydroxide (12): This was synthesized by the same procedure as described for $\mathbf{6}$, but using $\alpha$-Cl-DMA in place of $\alpha$-Cl-DEA. Yield $87 \%$. m.p. $>185^{\circ} \mathrm{C}$ (decomp). $\quad \mathrm{C}_{60} \mathrm{H}_{84} \mathrm{~N}_{4} \mathrm{O}_{8} \mathrm{NaOH} \cdot \mathrm{H}_{2} \mathrm{O}$ (1046.63): C 68.81, H 8.37, N 5.35, Na 2.20; found: C 68.53, H 8.53, N 5.68, Na 2.51\%. FTIR: $(\mathrm{KBr}$, $\left.\mathrm{cm}^{-1}\right)$ : $1657\left(v_{\mathrm{C}=\mathrm{O}}\right), 3425\left(v_{\mathrm{OH}}\right) \cdot{ }^{1} \mathrm{H}$ NMR: $\left(\mathrm{CDCl}_{3}, \delta\right.$ ppm): $7 \cdot 18(s, 8 \mathrm{H}, \mathrm{Ar}-\mathrm{H}), 4.63\left(s, 8 \mathrm{H}, \mathrm{OCH}_{2} \mathrm{CO}\right)$, $4.57\left(d, J=12.43 \mathrm{~Hz}, 4 \mathrm{H}, \mathrm{Ar}-\mathrm{CH}_{2}-\mathrm{Ar}\right), 3.30(d$, $\left.J=12.07 \mathrm{~Hz}, 4 \mathrm{H}, \mathrm{Ar}-\mathrm{CH}_{2}-\mathrm{Ar}\right), 3 \cdot 05,2.92(s, 12 \mathrm{H}$ each, $\left.\mathrm{NCH}_{3}\right), 1.18\left(s, 36 \mathrm{H}, \mathrm{C}\left(\mathrm{CH}_{3}\right)_{3}\right),{ }^{13} \mathrm{C}$ NMR: $\left(\mathrm{CDCl}_{3}, \quad \delta\right.$ ppm $): \quad 30.22 \quad\left(\mathrm{Ar}-\mathrm{CH}_{2}-\mathrm{Ar}\right), \quad 31.39$

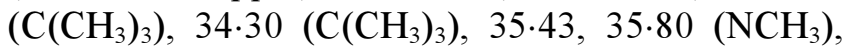
$73.93\left(\mathrm{OCH}_{2}\right), 125.82\left(\mathrm{C}_{m}\right), 134.96\left(\mathrm{C}_{o}\right), 148.17$ $\left(\mathrm{C}_{p}\right), 151.01\left(\mathrm{C}_{i}\right), 169.16(\mathrm{C}=\mathrm{O})$. FAB-MS: $m / z$ (intensity (\%), fragment) $1012\left(100,[\mathrm{M}]^{+}\right)$.

\section{$2.21 \quad[(5,11,17,23$-Tetra-tert-butyl-25, 26,27,28-tetra-}

kis (N,N-dimethylaminocarbonyl) methoxycalix [4] arene)potassium(I)]5,11,17,23-tetra-tert-butyl-25, 26, 27, 28-tetrahydroxycalix [4]arene-monoanion, (13): This was synthesized by the same procedure as described for 12, but using $\mathrm{K}_{2} \mathrm{CO}_{3}$ in place of Na$\mathrm{HCO}_{3}$. Yield $87 \%$. m.p. $>185^{\circ} \mathrm{C}$ (decomp). $\mathrm{C}_{104} \mathrm{H}_{140} \mathrm{~N}_{4} \mathrm{O}_{12} \mathrm{~K}$ (1676.01): C 74.47, H 8.41, N 3.34, K 2.33; Found: C 74.16, H 8.21, N 3.42, K 2.54\%. FTIR: $\left(\mathrm{KBr}, \mathrm{cm}^{-1}\right)$ : $1659\left(v_{\mathrm{C}=\mathrm{O}}\right), 3420\left(v_{\mathrm{OH}}\right) .{ }^{1} \mathrm{H}$ NMR: $\left(\mathrm{CDCl}_{3}, \delta \mathrm{ppm}\right): 7.18(s, 8 \mathrm{H}, \mathrm{Ar}-\mathrm{H}), 6.94(s, 8 \mathrm{H}$, $\mathrm{Ar}-\mathrm{H}), 4.63\left(s, 8 \mathrm{H}, \mathrm{OCH}_{2} \mathrm{CO}\right), 4.57(d, J=12.43 \mathrm{~Hz}$, $\left.4 \mathrm{H}, \mathrm{Ar}-\mathrm{CH}_{2}-\mathrm{Ar}\right), 4.51(d, J=12.06 \mathrm{~Hz}, 4 \mathrm{H}, \mathrm{Ar}-$ $\left.\mathrm{CH}_{2}-\mathrm{Ar}\right), 3.30\left(d, J=12.07 \mathrm{~Hz}, 4 \mathrm{H}, \mathrm{Ar}-\mathrm{CH}_{2}-\mathrm{Ar}\right)$, $3.19\left(d, J=12.22 \mathrm{~Hz}, 4 \mathrm{H}, \mathrm{Ar}-\mathrm{CH}_{2}-\mathrm{Ar}\right), 3.05,2.92$ $\left(s, 12 \mathrm{H}\right.$ each, $\left.\mathrm{NCH}_{3}\right), 1.18\left(m, 72 \mathrm{H}, \mathrm{C}\left(\mathrm{CH}_{3}\right)_{3}\right)$. ${ }^{13} \mathrm{C}$ NMR: $\left(\mathrm{CDCl}_{3}, \delta \mathrm{ppm}\right): 29.59,30.95\left(\mathrm{Ar}-\mathrm{CH}_{2}-\right.$

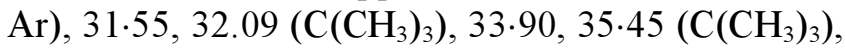
$35.74,36.01\left(\mathrm{NCH}_{3}\right), 72.03\left(\mathrm{OCH}_{2}\right), 125.44,125.90$ $\left(\mathrm{C}_{m}\right), 133.66,134.54\left(\mathrm{C}_{o}\right), 144.66,147.44\left(\mathrm{C}_{p}\right)$, $151.51,153.52\left(\mathrm{C}_{i}\right), 170 \cdot 14(\mathrm{C}=\mathrm{O})$. FAB-MS: $\mathrm{m} / \mathrm{z}$ (intensity (\%), fragment) 1029 (25, [M-calix[4]arenemonoanion $+\mathrm{H}]^{+}$).

$2.2 \mathrm{~m} \quad[(5,11,17,23-$ Tetra-tert-butyl-25,26,27,28-tetrakis(N,N-dimethylaminocarbonyl) methoxycalix [4]arene) potassium(I)]hydroxide, (14): This was synthesized by the same procedure as described for $\mathbf{1 2}$, but using $\mathrm{KHCO}_{3}$ in place of $\mathrm{NaHCO}_{3}$. Yield $80 \%$. m.p. $>200^{\circ} \mathrm{C}$ (decomp.). $\mathrm{C}_{60} \mathrm{H}_{84} \mathrm{~N}_{4} \mathrm{O}_{8} \mathrm{KOH} \cdot \mathrm{H}_{2} \mathrm{O}(1062 \cdot 26)$ : C 67.76 H 8.25, N 5.27, K 3.68; found: C 67.33, H 8.54, N 5.58, K 3.59\%. FTIR: $\left(\mathrm{KBr}, \mathrm{cm}^{-1}\right): 1654$ $\left(v_{\mathrm{C}=\mathrm{O}}\right), 3430\left(v_{\mathrm{OH}}\right) .{ }^{1} \mathrm{H}$ NMR: $\left(\mathrm{CDCl}_{3}, \delta \mathrm{ppm}\right): 7 \cdot 15$ $(s, 8 \mathrm{H}, \mathrm{Ar}-\mathrm{H}), 4.55\left(s, 8 \mathrm{H}, \mathrm{OCH}_{2} \mathrm{CO}\right), 4.49(d$, 

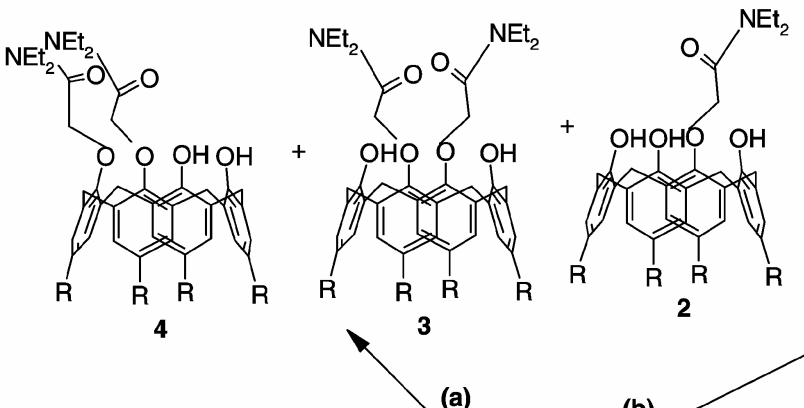

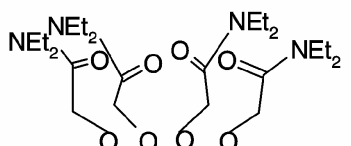
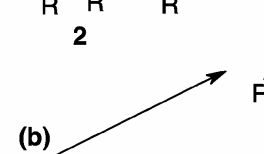

(b) 5

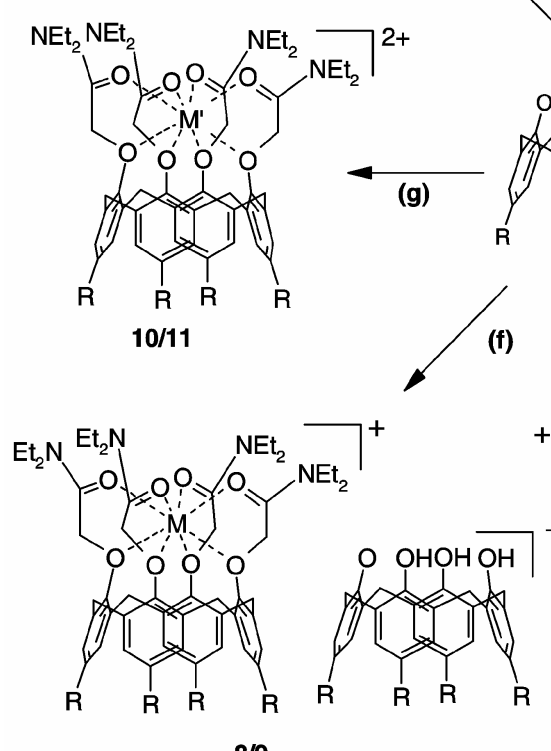

(a)
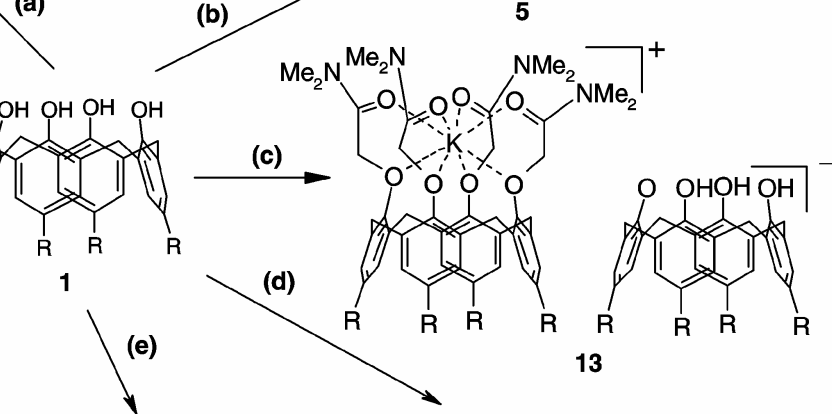

13

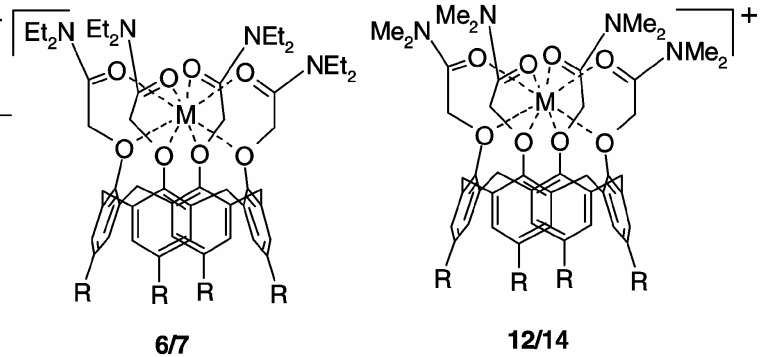

Scheme 1. Reactions of $p$-tert-butyl-calix[4]arene, 1 with $\alpha$-Cl-DEA or $\alpha$-Cl-DMA in the presence of alkali or alkaline earth metal salts (a) NaH, THF + DMF, $\alpha$-Cl-DEA $(1: 3: 3), 2 \mathrm{~h}$; (b) NaH, THF + DMF, $\alpha$-Cl-DEA (1:8:8), $8 \mathrm{~h}$; (c) $\mathrm{K}_{2} \mathrm{CO}_{3}, \mathrm{CH}_{3} \mathrm{CN}, \alpha$-Cl-DMA (1:5:5), $36 \mathrm{~h}$; (d) $\mathrm{Na}_{2} \mathrm{CO}_{3}$ or $\mathrm{MHCO}_{3}$, $\mathrm{CH}_{3} \mathrm{CN}, \alpha$-Cl-DMA $(1: 5: 5), 36 \mathrm{~h}$; (e) $\mathrm{MOH}$ or $\mathrm{MHCO}_{3}, \mathrm{CH}_{3} \mathrm{CN}, \alpha$-Cl-DEA (1:5:5), $36 \mathrm{~h}$; (f) $\mathrm{M}_{2} \mathrm{CO}_{3}$, $\mathrm{CH}_{3} \mathrm{CN}, \alpha$-Cl-DEA $(1: 5: 5), 36 \mathrm{~h}$; $(\mathrm{g}) \mathrm{CaH}_{2}$ or $\mathrm{M}^{\prime}(\mathrm{OH})_{2}, \mathrm{CH}_{3} \mathrm{CN}, \alpha$-Cl-DEA $(1: 5: 5), 36 \mathrm{~h} . \mathrm{M}=\mathrm{Na}^{+}$ $(6,8,12)$ and $\mathrm{K}^{+}(7,9,13,14) ; \mathrm{M}^{\prime}=\mathrm{Ca}^{2+}(\mathbf{1 0})$ and $\mathrm{Sr}^{2+}(\mathbf{1 1})$. The ratios given here corresponds to, 1 : base: $\alpha-\mathrm{Cl}-\mathrm{DEA} / \alpha-\mathrm{Cl}-\mathrm{DMA}$. All the reactions were carried out at refluxing conditions. Please refer to the table 1 in order to note all the products formed in the reaction.

$\left.J=12.05,4 \mathrm{H}, \mathrm{Ar}-\mathrm{CH}_{2}-\mathrm{Ar}\right), 3 \cdot 35(d, J=12 \cdot 05 \mathrm{~Hz}$, $\left.4 \mathrm{H}, \mathrm{Ar}-\mathrm{CH}_{2}-\mathrm{Ar}\right), 3 \cdot 00,2 \cdot 94\left(\mathrm{~s}, 12 \mathrm{H}\right.$ each, $\left.\mathrm{NCH}_{3}\right), 1 \cdot 18$ $\left(s, 36 \mathrm{H}, \mathrm{C}\left(\mathrm{CH}_{3}\right)_{3}\right) .{ }^{13} \mathrm{C} \mathrm{NMR}:\left(\mathrm{CDCl}_{3}, \delta \mathrm{ppm}\right): 30 \cdot 25$

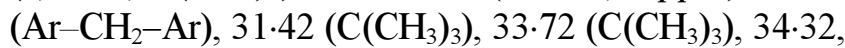
$35.45\left(\mathrm{NCH}_{3}\right), 73.94\left(\mathrm{OCH}_{2}\right), 125.91\left(C_{\mathrm{m}}\right), 135.02$ $\left(\mathrm{C}_{o}\right), 148 \cdot 34\left(\mathrm{C}_{p}\right), 151 \cdot 10\left(\mathrm{C}_{i}\right), 169 \cdot 11(\mathrm{C}=\mathrm{O}) \mathrm{FAB}-$ MS: $m / z$ (intensity $(\%)$, fragment) $1028\left(100,[\mathrm{M}]^{+}\right)$.

\section{Results and discussion}

3.1 Role of alkali metal ions on the reaction between $p$-tert-butyl-calix[4]arene and $\alpha$-chlorodiethylacetamide ( $\alpha$-Cl-DEA)

Reactions between the p-tert-butylcalix[4]arene, 1 and $\alpha$-Cl-DEA were carried out in the presence of carbonates, bicarbonates and hydroxides of $\mathrm{Li}^{+}, \mathrm{Na}^{+}$, $\mathrm{K}^{+}, \mathrm{Rb}^{+}$and $\mathrm{Cs}^{+}$in acetonitrile under $\mathrm{N}_{2}$ atmosphere (scheme 1). The reactions of 1 and $\alpha$-Cl-DEA in the presence of $\mathrm{Li}^{+}$salts yield no product and only the unreacted calix[4]arene was recovered even when the reaction periods were long (table 1).

Reaction of 1 and $\alpha$-Cl-DEA in the presence of $\mathrm{Na}_{2} \mathrm{CO}_{3}$ in the ratio $1: 2: 2$ for $20 \mathrm{~h}$ yielded monoamide derivative (2) along with some unreacted 1 (table 1). However, when the same reaction was performed at $1: 5: 5$ for a period of $36 \mathrm{~h}$, a product mixture containing mono- (2), 1,3-di- (3) amide derivatives in addition to $\mathbf{8}$ were formed. Product $\mathbf{8}$ was separated from this mixture by fractional crystallization, and $\mathbf{2}$ and $\mathbf{3}$ were separated by chromatography. On the basis of ${ }^{1} \mathrm{H}$ and ${ }^{13} \mathrm{C}$ NMR spectra, 
Table 1. Reactions carried out between $\mathbf{1}$ and $\alpha$-Cl-DEA in the presence of alkali and alkaline earth salts and the products obtained."

\begin{tabular}{|c|c|c|c|c|}
\hline $\begin{array}{l}\text { Entry } \\
\text { number }\end{array}$ & Base & $1:$ Base: $\alpha$-Cl-DEA & Reaction time (h) & Product(s) (yield, \%) \\
\hline 1 & $\mathrm{Li}_{2} \mathrm{CO}_{3}$ & $1: 2: 2 ; 1: 5: 5$ & 20,36 & No product \\
\hline 2 & $\mathrm{Na}_{2} \mathrm{CO}_{3}$ & $1: 2: 2^{*}$ & 20 & $2(20)$ \\
\hline 3 & & $1: 5: 5$ & 36 & $\mathbf{2}(10), \mathbf{3}(15), \mathbf{8}(25)$ \\
\hline 4 & $\mathrm{~K}_{2} \mathrm{CO}_{3}$ & $1: 2: 2 ; 1: 5: 5$ & $5,10,20$ or 36 & $9(90)$ \\
\hline 5 & $\mathrm{Rb}_{2} \mathrm{CO}_{3}$ & $1: 2: 2^{*}$ & 20 & $2(15)$ \\
\hline 6 & & $1: 5: 5$ & 36 & $2(15), 3(23)$ \\
\hline 7 & $\mathrm{Cs}_{2} \mathrm{CO}_{3}$ & $1: 2: 2^{*}$ & 20 & $2(18)$ \\
\hline 8 & & $1: 5: 5$ & 36 & $5(55)$ \\
\hline 9 & $\mathrm{NaHCO}_{3}$ & $1: 2: 2$ & 20 & $3(10), 6(27)$ \\
\hline 10 & & $1: 5: 5$ & 36 & $6(80)$ \\
\hline 11 & $\mathrm{KHCO}_{3}$ & $1: 2: 2^{*}$ & 20 & $2(12), 7(35)$ \\
\hline 12 & & $1: 5: 5$ & 36 & $7(85)$ \\
\hline 13 & $\mathrm{RbHCO}_{3}$ & $1: 2: 2^{*}$ & 36 & $3(15)$ \\
\hline 14 & & $1: 5: 5^{*}$ & 36 & $3(35)$ \\
\hline 15 & $\mathrm{CsHCO}_{3}$ & $1: 3: 3$ & 36 & $3(80)$ \\
\hline 16 & & $1: 5: 5$ & 36 & $3(40), 5(30)$ \\
\hline 17 & $\mathrm{LiOH}$ & $1: 2: 2 ; 1: 5: 5$ & 20,36 & No product \\
\hline 18 & $\mathrm{NaOH}$ & $1: 2: 2^{*}$ & 20 & $\mathbf{2}(10), \mathbf{3}(25), \mathbf{6}(8)$ \\
\hline 19 & & $1: 5: 5$ & 36 & $6(82)$ \\
\hline 20 & $\mathrm{KOH}$ & $1: 2: 2^{*}$ & 20 & $\mathbf{2}(10), \mathbf{3}(25), 7(10)$ \\
\hline 21 & & $1: 5: 5$ & 36 & $7(75)$ \\
\hline 22 & $\mathrm{RbOH}^{*}$ & $1: 2: 2$ & 20 & $3(25)$ \\
\hline 23 & & $1: 5: 5$ & 36 & $3(25), 5(35)$ \\
\hline 24 & $\mathrm{CsOH}^{*}$ & $1: 2: 2$ & 20 & $3(30)$ \\
\hline 25 & & $1: 5: 5$ & 36 & $3(25), 5(50)$ \\
\hline 26 & $\mathrm{NaH}$ & $1: 3: 3$ & 2 & $2(15), 3(55), 4(5)$ \\
\hline 27 & & $1: 8: 8$ & 8 & $5(95)$ \\
\hline 28 & $\mathrm{MgCO}_{3} *$ & $1: 2: 2,1: 5: 5$ & 20,36 & No product \\
\hline 29 & $\mathrm{CaCO}_{3} *$ & $1: 5: 5,1: 10: 10$ & 20,36 & No product \\
\hline 30 & $\mathrm{SrCO}_{3} *$ & $1: 5: 5$ & 20,36 & No product \\
\hline 31 & $\mathrm{Mg}(\mathrm{OH})_{2} *$ & $1: 5: 5$ & 36 & No product \\
\hline 32 & $\mathrm{Ca}(\mathrm{OH})_{2}$ & $1: 2: 2^{*}$ & 36 & $2(15), 10(25)$ \\
\hline 33 & & $1: 5: 5$ & 36 & $10(77)$ \\
\hline 34 & $\mathrm{Sr}(\mathrm{OH})_{2}$ & $1: 2: 2^{*}$ & 20 & $\mathbf{2}(5), \mathbf{5}(10), \mathbf{1 1}(15)$ \\
\hline 35 & & $1: 5: 5$ & 36 & $11(90)^{-}$ \\
\hline 36 & $\mathrm{Ba}(\mathrm{OH})_{2}$ & $1: 2: 2^{*}$ & 20 & $5(20)$ \\
\hline 37 & & $1: 5: 5$ & 36 & $5(77)$ \\
\hline 38 & $\mathrm{CaH}_{2}$ & $1: 5: 5$ & 36 & $10(84)$ \\
\hline
\end{tabular}

${ }^{\#}$ Refer to the Scheme 1 for identity of products. *Unreacted calix[4]arene is present

8 was identified to be $\mathrm{Na}^{+}$bound calix[4] arene-tetramide where an additional calix[4]arene acts as counter anion. Similar reaction between 1 and $\alpha$-Cl-DEA in the presence $\mathrm{K}_{2} \mathrm{CO}_{3}$ in $1: 2: 2$ ratio for $20 \mathrm{~h}$ yielded a single product 9 that is analogous to 8 but $\mathrm{K}^{+}$is present in place of $\mathrm{Na}^{+}$and the structure of 9 was determined by single crystal Xray diffraction as reported in this paper. The same product was obtained even when this reaction was repeated with $1: 3: 3,1: 3: 5$ and $1: 5: 5$ ratios indicating that the charge of the monoanion of $p$-tertbutyl-calix[4]arene is counter-balanced by the $\mathrm{K}^{+}$- bound tetra-amide derivative, where the reaction is driven by the template action of $\mathrm{K}^{+}$. However, $1: 5: 5$ reaction carried out using either $\mathrm{MHCO}_{3}$ or $\mathrm{MOH}(\mathrm{M}=\mathrm{Na}$ or $\mathrm{K})$ resulted in a single product, 3 or 6 or $7, \mathrm{a} \mathrm{M}^{+}$bound calix[4]arene-tetramide where the counter anion could not be identified based on ${ }^{1} \mathrm{H}$ NMR and hence rules out the presence of a calixarene based anion. On the other hand, FTIR spectra suggest the presence of $\mathrm{OH}^{-}$species. The molecular ion peak in FAB mass spectra of $\mathbf{6}$ and 7 corresponds to a tetra-amide derivative bound to $\mathrm{M}^{+}$. Thus, the mono-anion of calix[4]arene was formed only in the 
case of carbonate and not with other anions and hence an anion dependency.

On the other hand, the reactions carried out in the presence of carbonate, bicarbonate and hydroxide salts of $\mathrm{Rb}^{+}$and $\mathrm{Cs}^{+}$always yielded a mixture of products including the unreacted 1, whether these were carried out at lower or at higher ratios (table 1), with an exception noticed in case of $\mathrm{CsHCO}_{3}$. Reaction between 1 and $\alpha$-Cl-DEA in the presence of $\mathrm{CsHCO}_{3}$ yielded a mixture of 1,3-di-(3) and tetra(5) amide derivatives at $1: 5: 5$ ratio and only a single product, viz. 1,3-di-amide (3) derivative at $1: 3: 3$ ratio. In the literature, ${ }^{20}$ it was reported to have been obtained 3 alone when the reaction was carried out in the presence of $\mathrm{K}_{2} \mathrm{CO}_{3}$ in $1: 1: 2$ ratio. However, the same reaction in our hands yielded a mixture of mono- (2) and 1,3-di- (3) -amide derivatives instead of the claimed 3 alone. Therefore, the formation of 1,3-di-amide derivative, 3 in the reaction of $\mathrm{CsHCO}_{3}$ at $1: 3: 3$ is interesting and important as the same derivative has been synthesized in the literature by going through four-steps. ${ }^{17}$ The literature procedure has several disadvantages besides the number of steps and effective low yields, such as, long reaction periods (4-5 days) and handling of corrosive (thionylchloride), carcinogenic (benzene) and unstable (diacid-chloride) substances or species. However, the procedure reported in this paper yields a pure product in as high as $80 \%$ yield in a singlestep without the use of such hazardous chemicals and in just 1.5 days.

Reaction between 1 and $\alpha$-Cl-DEA in presence of $\mathrm{NaH}$ in THF/DMF in the ratio of $1: 3: 3$ yielded a product $^{21}$ mixture of mono- (2), 1,3-di- (3) and 1,2di- (4) amide derivatives (scheme 1 and table 1) as confirmed based on ${ }^{1} \mathrm{H}$ NMR. However, when the same reaction was carried out at a higher ratio, viz. $1: 8: 8$, only a single product, calix[4]arene-tetraamide (5) was obtained and this result is consistent with that reported in the literature. ${ }^{22}$ Thus, among all the reactions reported in this paper only the $\mathrm{NaH}$ reaction leads to the formation of the 1,2-di-amide derivative.

\subsection{Role of alkaline earth metal ions on the reaction between p-tert-butyl-calix[4]arene and $\alpha-C l-D E A$}

While alkali carbonates were found to be active in these $O$-alkylation reactions (table 1) that of alkaline earth ones, $\mathrm{Mg}^{2+}, \mathrm{Ca}^{2+}$ and $\mathrm{Sr}^{2+}$ were inactive either at $1: 2: 2$ or at $1: 5: 5$ ratios. Similar is the case with the reactions of $\operatorname{Mg}(\mathrm{OH})_{2}$. The reactions of $\mathrm{CaH}_{2}$, and the hydroxides of $\mathrm{Ca}^{2+}$ and $\mathrm{Sr}^{2+}$ (diagonally related to $\mathrm{Na}^{+}$and $\mathrm{K}^{+}$) resulted in the formation of $\mathrm{M}^{2+}$ bound tetra-amide derivatives, viz. 10 and 11 which are similar to $6\left(\mathrm{Na}^{+}\right)$and $7\left(\mathrm{~K}^{+}\right)$but the bound metal ion is different. On the other hand, the $\mathrm{Ba}(\mathrm{OH})_{2}$ reaction resulted in pure tetra-amide derivative (5) in high yields. The other high yield method reported in the literature ${ }^{22}$ for synthesizing tetraamide is the reaction using $\mathrm{NaH}$. Thus, the demonstration of the use of $\mathrm{Ba}(\mathrm{OH})_{2}$ in making tetra-amide derivative in high yield is certainly adventitious as the corresponding synthetic procedure does not demand for handling $\mathrm{NaH}$. Thus, it is evident from these results that among the alkaline earth metal ions, only $\mathrm{Ca}^{2+}$ and $\mathrm{Sr}^{2+}$ hydroxides were found to act as templates for the formation of $\mathrm{M}^{2+}$ bound calix[4]arene-tetraamide derivatives.

\subsection{Role of alkali metal ions on the reaction \\ between p-tert-butyl-calix[4]arene and $\alpha$-chlorodimethylacetamide ( $\alpha$-Cl-DMA)}

Reaction between 1 and $\alpha$-Cl-DMA in the presence of $\mathrm{Na}_{2} \mathrm{CO}_{3}$ and $\mathrm{K}_{2} \mathrm{CO}_{3}(1: 5: 5$ ratio and $36 \mathrm{~h}$, reflux) yielded 12 and 13 respectively in $85 \%$ yield. These are analogous to 6 and 9 obtained in case of $\alpha-\mathrm{Cl}$ DEA reaction. Under similar reaction conditions, $\mathrm{NaHCO}_{3}$ and $\mathrm{KHCO}_{3}$ gave compounds, 12 and 14 respectively in about $85 \%$ yield. These products are analogous to 6 and 7 obtained in case of $\alpha$-Cl-DEA reaction, viz. $\mathrm{M}^{+}$bound tetra-amide derivative. Thus, the change in amide precursor from $\alpha$-Cl-DEA to $\alpha$ Cl-DMA did not bring any change in the type of amide derivative formed.

\subsection{Separation of individual products and characterization}

As can be seen from the table 1 that many reactions yield a mixture of products. TLC and ${ }^{1} \mathrm{H}$ NMR studies clearly indicated the presence of different products in these mixtures. However, the individual products were separated subsequently and were characterized by ${ }^{1} \mathrm{H}$ and ${ }^{13} \mathrm{C}$ NMR and FAB mass spectra. All the separated products have clearly shown satisfactory elemental and metal ion analyses. FTIR, NMR, MS and elemental data of all the compounds are presented in the experimental section. 
3.4a Identification of different amide derivatives: ${ }^{1} \mathrm{H}$ and ${ }^{13} \mathrm{C}$ NMR spectra were measured in $\mathrm{CDCl}_{3}$ for all the products. The spectra were consistent with the presence of cone conformation as deduced based on the bridged methylene group resonances.
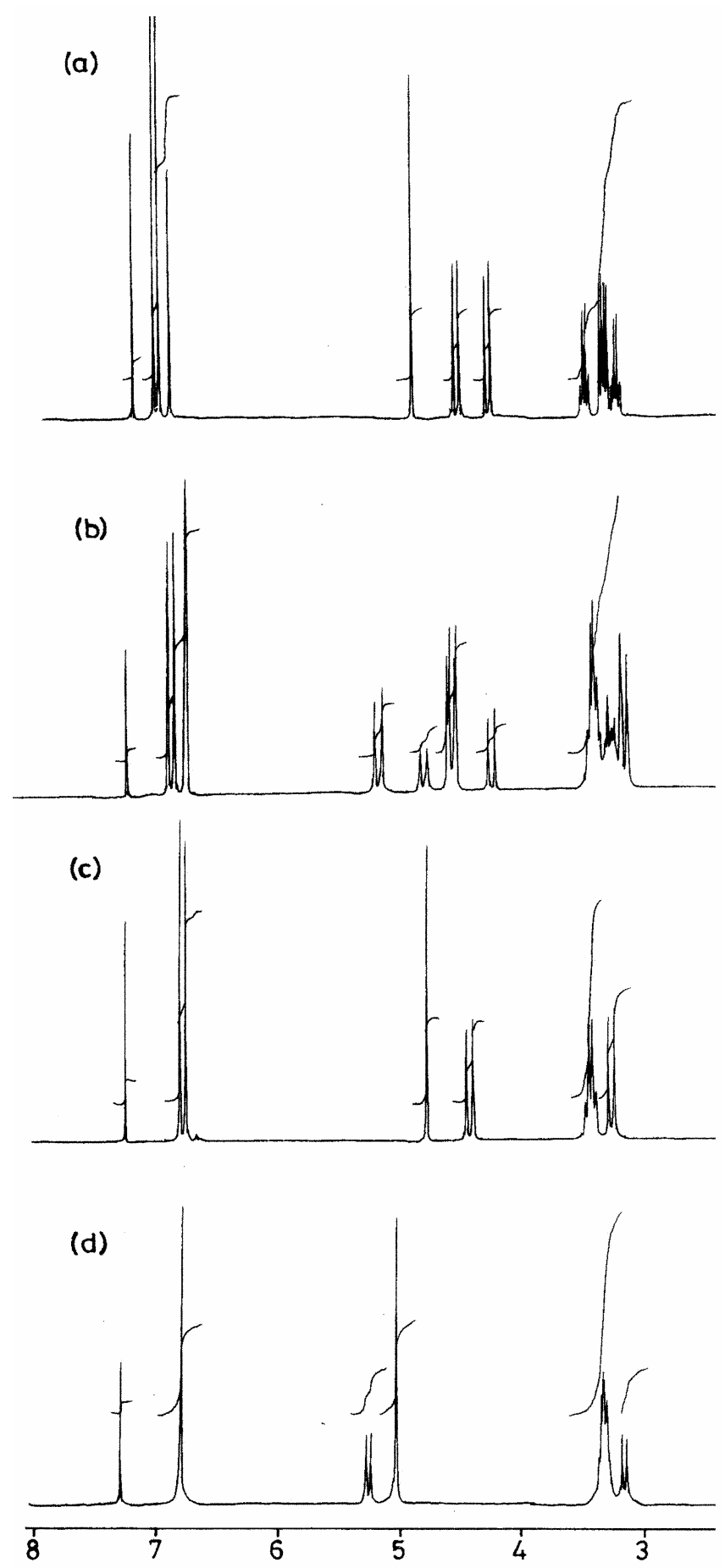

Figure 1. Comparison among ${ }^{1} \mathrm{H}$ NMR spectra of various calix[4]arene-amide derivatives: (a) 2 (mono); (b) 3 (1,3-di); (c) 4 (1,2-di) and (d) 5 (tetra).
Two multiplets appeared at 1.05 and $3.33 \mathrm{ppm}$ arising from methyl and methylene protons of $-\mathrm{NCH}_{2} \mathrm{CH}_{3}$ moiety respectively supported the presence of amide arms in these derivatives. The different calix[4] arene-amide derivatives can be easily differentiated based on $-\mathrm{OCH}_{2}$ signals, viz. 4.96 (mono-), 4.67 (doublet, 1,2-di-), 4.82 (1,3-di-) and 5.02 (tetraamide) as well as the aromatic signals, viz. mono(three singlets), 1,2-di- (two doublets and one doublet of doublet), 1,3-di- (two singlets) and tetra- (one singlet) as can be seen from figure 1 . In case of the spectra measured from the product mixture, the peak areas will provide an estimate of the amount of products formed.

3.4b Identification of metal ion bound species: In FTIR, $v_{\mathrm{CO}}$ bands did not show any considerable shift in the tetra-amide bound to $\mathrm{Na}^{+}\left(6\right.$ and 8) or $\mathrm{K}^{+}(7$ and 9) when compared to the corresponding bands in free derivative and hence cannot be used for identifying $\mathrm{M}^{+}$binding. Changes observed in the $v_{\mathrm{OH}}$ are reflective of the presence of $\mathrm{OH}^{-}$counter ion in case of 8 and 9. On the other hand, $\mathrm{Ca}^{2+}(\mathbf{1 0})$ and $\mathrm{Sr}^{2+}$

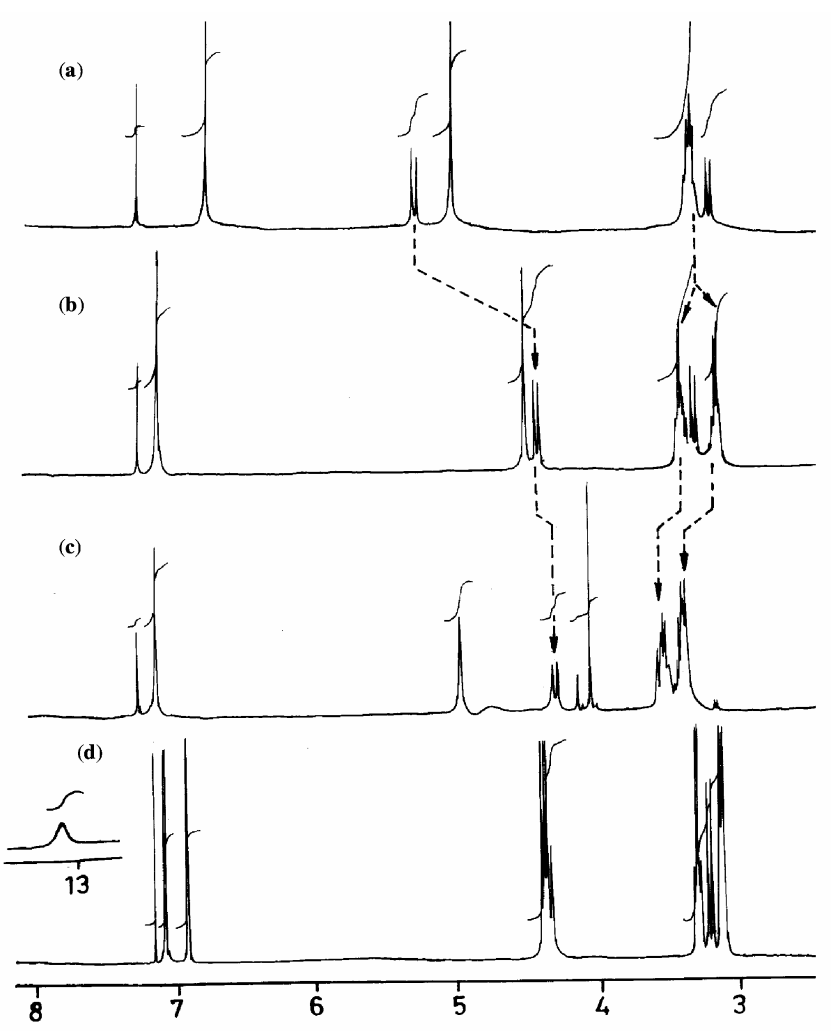

Figure 2. Comparison among ${ }^{1} \mathrm{H}$ NMR spectra of calix[4]arene-tertra-amide (a) 5; and its complexes, (b) 7 $\left(\mathrm{K}^{+}\right)$; (c) $10\left(\mathrm{Ca}^{2+}\right)$ and (d) $9\left(\mathrm{~K}^{+}\right.$, along with calix [4] arene-monoanion). 
Table 2. Crystal structure refinement parameters for 9.

\begin{tabular}{ll}
\hline Empirical formula & $\mathrm{C}_{119} \mathrm{H}_{165 \cdot 5} \mathrm{KN}_{7 \cdot 5} \mathrm{O}_{12}$ \\
Formula weight & $1930 \cdot 68$ \\
Temperature & $150(2) \mathrm{K}$ \\
Wavelength & $0 \cdot 71073 \AA$ \\
Crystal system & Monoclinic \\
Space group & $P_{n}$ \\
Unit cell dimensions & $a=16 \cdot 300(5)$ \\
& $b=21 \cdot 518(5)$ \\
& $c=16 \cdot 776(5) \AA$ \\
Volume & $\beta=102 \cdot 76(2)^{\circ}$ \\
$Z$ & $5739(3) \AA^{3}$ \\
Density (calculated) & 2 \\
Absorption coefficient & $1 \cdot 117 \mathrm{mg} / \mathrm{m}^{3}$ \\
$F$ (000) & $0 \cdot 107 \mathrm{~mm}$ \\
Crystal size & 2091 \\
Theta range for data collection & $0 \cdot 30 \times 0 \cdot 10 \times 0 \cdot 10 \mathrm{~mm}^{3}$ \\
Index ranges & $3 \cdot 46$ to $26 \cdot 37^{\circ}$ \\
Reflections collected & $-20 \leq h \leq 20,-26 \leq k \leq 26,-20 \leq 1 \leq 20$ \\
Independent reflections & 44101 \\
Completeness to theta & $23060[R(\mathrm{int})=0 \cdot 0267]$ \\
Absorption correction & $26 \cdot 37^{\circ}(99 \cdot 7 \%)$ \\
Max. and min. transmission & $\mathrm{Semi}-\mathrm{empirical}$ \\
Data/restraints/parameters & $0 \cdot 9894$ and $0 \cdot 9687$ \\
Goodness-of-fit on $F^{2}$ & $23060 / 2 / 1293$ \\
Final $R$ indices $[I>2$ sigma $(I)]$ & $1 \cdot 032$ \\
$R$ indices (all data) & $R 1=0 \cdot 0438, w R 2=0 \cdot 1071$ \\
Extinction coefficient & $R 1=0 \cdot 0533, w R 2=0 \cdot 1132$ \\
Largest diff. peak and hole & $0 \cdot 0049(4)$ \\
\hline & $0 \cdot 456$ and $-0 \cdot 340 \mathrm{e} \AA^{-3}$ \\
&
\end{tabular}

Table 3. Selected bond lengths $(\AA)$ and bond angles $\left({ }^{\circ}\right)$ of $\mathrm{K}^{+}$coordination sphere in 9.

\begin{tabular}{llll}
\hline $\mathrm{K}(1)-\mathrm{O}(8)$ & $2 \cdot 637(2)$ & $\mathrm{K}(1)-\mathrm{O}(7)$ & $2 \cdot 698(2)$ \\
$\mathrm{K}(1)-\mathrm{O}(10)$ & $2 \cdot 637(2)$ & $\mathrm{K}(1)-\mathrm{O}(12)$ & $2 \cdot 710(2)$ \\
$\mathrm{K}(1)-\mathrm{O}(5)$ & $2 \cdot 664(2)$ & $\mathrm{K}(1)-\mathrm{O}(9)$ & $2 \cdot 717(2)$ \\
$\mathrm{K}(1)-\mathrm{O}(6)$ & $2 \cdot 691(2)$ & $\mathrm{K}(1)-\mathrm{O}(11)$ & $2 \cdot 813(2)$ \\
$\mathrm{O}(8)-\mathrm{K}(1)-\mathrm{O}(10)$ & $126 \cdot 3(1)$ & $\mathrm{O}(6)-\mathrm{K}(1)-\mathrm{O}(9)$ & $130 \cdot 7(1)$ \\
$\mathrm{O}(8)-\mathrm{K}(1)-\mathrm{O}(5)$ & $79 \cdot 3(1)$ & $\mathrm{O}(7)-\mathrm{K}(1)-\mathrm{O}(9)$ & $148 \cdot 0(1)$ \\
$\mathrm{O}(10)-\mathrm{K}(1)-\mathrm{O}(5)$ & $60 \cdot 5(1)$ & $\mathrm{O}(12)-\mathrm{K}(1)-\mathrm{O}(9)$ & $136 \cdot 6(1)$ \\
$\mathrm{O}(8)-\mathrm{K}(1)-\mathrm{O}(6)$ & $77 \cdot 1(1)$ & $\mathrm{O}(8)-\mathrm{K}(1)-\mathrm{O}(11)$ & $71 \cdot 7(1)$ \\
$\mathrm{O}(10)-\mathrm{K}(1)-\mathrm{O}(6)$ & $154 \cdot 3(1)$ & $\mathrm{O}(10)-\mathrm{K}(1)-\mathrm{O}(11)$ & $134 \cdot 5(1)$ \\
$\mathrm{O}(5)-\mathrm{K}(1)-\mathrm{O}(6)$ & $123 \cdot 1(1)$ & $\mathrm{O}(5)-\mathrm{K}(1)-\mathrm{O}(11)$ & $150 \cdot 1(1)$ \\
$\mathrm{O}(8)-\mathrm{K}(1)-\mathrm{O}(7)$ & $123 \cdot 4(1)$ & $\mathrm{O}(6)-\mathrm{K}(1)-\mathrm{O}(11)$ & $57 \cdot 3(1)$ \\
$\mathrm{O}(10)-\mathrm{K}(1)-\mathrm{O}(7)$ & $82 \cdot 8(1)$ & $\mathrm{O}(7)-\mathrm{K}(1)-\mathrm{O}(11)$ & $125 \cdot 0(1)$ \\
$\mathrm{O}(10)-\mathrm{K}(1)-\mathrm{O}(9)$ & $74 \cdot 9(1)$ & $\mathrm{O}(12)-\mathrm{K}(1)-\mathrm{O}(11)$ & $85 \cdot 2(1)$ \\
$\mathrm{O}(5)-\mathrm{K}(1)-\mathrm{O}(9)$ & $71 \cdot 8(1)$ & $\mathrm{O}(9)-\mathrm{K}(1)-\mathrm{O}(11)$ & $86 \cdot 8(1)$ \\
\hline
\end{tabular}

(11) bound tetra-amide derivatives have shown a shift in the carbonyl stretching frequency by about 29 and $24 \mathrm{~cm}^{-1}$ respectively. However, ${ }^{1} \mathrm{H}$ NMR spectra exhibited clear-cut differences among the tetra-amide (5) and its corresponding metal ion complexes $(6,7,8,9,10$ and 11$)$ in the signals of bridged methylene and methylene of -NEt as shown in figure 2. In 8 and 9, where the calix[4]arenemonoanion is present, the spectra showed two sets of calix[4]arene based signals where one set arises from metal bound tetra-amide and the other from calix[4]arene-mono-anion. 


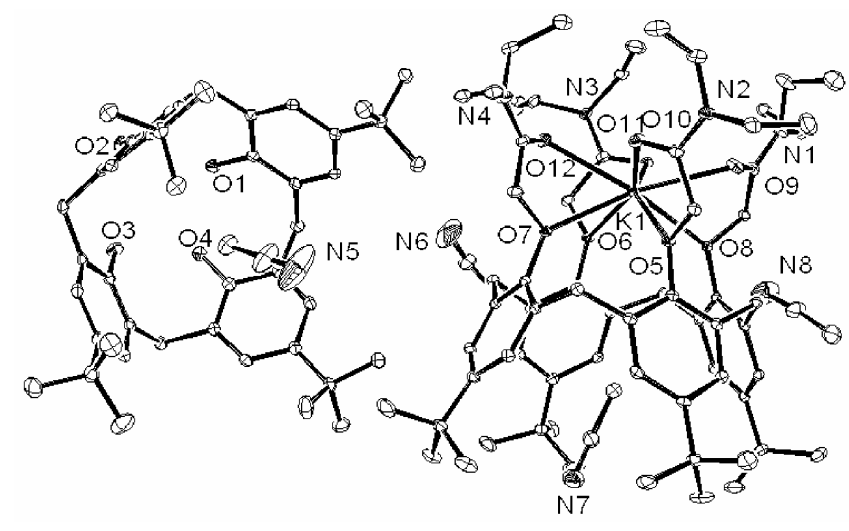

Figure 3. Crystal structure of 9 as ORTEP plot. The hetero-atoms are labelled for clarity. There are four acetonitrile molecules per unit cell.

\subsection{Crystal structure of 9}

The data collection and refinement parameters for the crystal structure, 9 were listed in table 2 . The molecular structure of 9 shown in figure 3 clearly indicates the presence two calix[4]arene units, of which one is an anionic and the other is $\mathrm{K}^{+}$bound tetraamide derivative. Important bond lengths and bond angles of the coordination sphere are summarized in table 3. In the cationic portion of the crystal structure of 9 , the $p$-tert-butyl-calix[4]arene is alkylated at all the four phenolic- $O$-centers by $\mathrm{N}, \mathrm{N}$-diethylacetamide moiety to result in a tetraamide derivative. The $\mathrm{K}^{+}$ion is bound through eight oxygens of the tetra-amide, of which four were from the amide moieties and the remaining were from the lower rim pendant ether oxygens. Thus, the complex shows bond distances of $\mathrm{K} \ldots \mathrm{O}_{\text {ether }}, 2.637-2.698 \AA$ and $\mathrm{K} \ldots \mathrm{O}_{\text {amide, }}, 2.637-2.813 \AA$ respectively. The geometry around $\mathrm{K}^{+}$fits well with a bi-capped octahedron. The counter anion in 9 exhibited a $\mathrm{O} \ldots \mathrm{O}(1,2-$ type) distances of $2.523,2.538,2.766$ and 2.949 , and $\mathrm{O} \ldots \mathrm{O}$ (1,3-type) distances of 3.610 and 3.936 consistent with a localized anion on the calix[4] arene moiety in the crystal structure and this data is comparable with the results reported in the literature. ${ }^{23}$ Also comparison of this data with that found in case of the neutral p-tert-butyl-calix[4]arene structure $^{24}$ supports the presence of mono-anion in case of 9 .

There are four acetonitrile molecules present in the unit cell, of which, one was located close to the lower rim oxygens of the anion with partly being buried in the hydrophobic environment, another was completely buried in the cavity of calixarene of the cationic part and the remaining two were in the lattice. On the other hand, the $\mathrm{K}^{+}$-complex of same $p$ tert-butyl-calix[4]arene-tetraamide with thiocyante or iodide counter anion was demonstrated where the pre-formed $p$-tert-butyl-calix[4]arene-tetraamide was simply reacted with the corresponding potassium salt, ${ }^{21}$ viz. KSCN or KI and was not obtained from the reactions of the type reported in this paper. Thus, in the literature, the $\mathrm{K}^{+}$bound tetra-amide structure is known, and also known is the structure of the calix[4]arene anion but not these two together in the same compound. Thus, 9 reported in this paper is altogether unknown in the literature and the crystallographic characterization is definitely done for the first time as reported in this paper.

\section{Conclusions}

This paper clearly demonstrates the interactive role of alkali and alkaline earth salts on the formation of calix[4]arene-amide derivatives in general and template action of $\mathrm{K}^{+}$in particular, through $O$-alkylation at the lower rim. Among the alkali and alkaline earth ions studied, $\mathrm{Li}^{+}$and $\mathrm{Mg}^{2+}$ were completely nonreactive.

The $\mathrm{Na}^{+}$and $\mathrm{K}^{+}$drives the reaction to form their metal ion bound tetra-amide derivatives, so is the case with $\mathrm{Ca}^{2+}$ and $\mathrm{Sr}^{2+}$ owing to their diagonal relationship. Though the carbonates, hydroxides and hydrides of alkali salts are capable of producing metal ion bound tetra-amide, it is the carbonate only that drives the formation of calix[4]arene-monoanion. While $\mathrm{Na}_{2} \mathrm{CO}_{3}$ produces this compound along with other products, $\mathrm{K}_{2} \mathrm{CO}_{3}$ provides this as a single product. On the other hand, none of the $\mathrm{Ca}^{2+}$ and $\mathrm{Sr}^{2+}$ show any calix[4]arene-monoanion formation. Therefore, it is $\mathrm{K}^{+}$that acts as template in these reactions.

The larger ions such as $\mathrm{Cs}^{+}$and $\mathrm{Ba}^{2+}$ can only drive the reaction to the formation of tetra-amide but not to the metal ion bound ones, indicating the inability of these ions to act as templates owing to the size constraints posed by the binding core formed from the four pendants of the tetra-amide derivative. Hence the template action is dependent on the size of the ion as well. Thus, among all the salts studied, it is the $\mathrm{K}_{2} \mathrm{CO}_{3}$ that produces the $\mathrm{K}^{+}$bound tetraamide while forming the calix[4]arene anion and no other combination could do this. Thus very small and very large ions were not effective in generating calix[4]arene monoanion along with the metal ion bound tetra-amide derivative. 
1,3-Di-amide was reported to have been synthesized in the literature via four-steps with long reaction period where corrosive, carcinogenic and unstable species have to be handled. However, the present paper demonstrates a straight-forward onestep reaction that gives high yield with low reaction period using $\mathrm{CsHCO}_{3}$.

Almost all the reactions carried out at a lower ratio $(1: 2: 2)$ yielded mono-amide derivative. Among all the reactions reported in this paper only the $\mathrm{NaH}$ reaction leads to the 1,2-di-amide derivative. None of the reactions reported in this paper yield tri-amide derivative. In order to synthesize tri-amide derivative a mixture of $\mathrm{BaO}$ and $\mathrm{Ba}(\mathrm{OH})_{2}$ were used. ${ }^{16}$

The change in amide precursor, viz. $\alpha$-Cl-DEA or $\alpha$-Cl-DMA, has no effect on the type of amide derivatives formed and hence suggests the general nature of the reactions demonstrated in this paper.

\section{Acknowledgements}

CPR acknowledges the financial support from the Council of Scientific and Industrial Research (CSIR) and Department of Science and Technology (DST), New Delhi, and Department of Atomic Energy (DAE). Central Drug Research Institute (CDRI), Lucknow is acknowledged for FAB mass spectra. AA acknowledges the SRF fellowship from CSIR.

\section{References}

1. Arduini A, Giorgi G, Pochini A, Secchi A and Ugozzoli F 2001 Tetrahedron 572411

2. Beer P D, Drew M G B, Gale P A, Leeson P B and Ogden M I 1994 J. Chem. Soc., Dalton Trans. 3479

3. Wolf N J, Georgiev E M, Yordanov A T, Whittlesey B R, Koch H F and Roundhill D M 1999 Polyhedron 18885

4. Moser A, Yap G P A and Detellier C 2002 J. Chem. Soc., Dalton Trans. 428

5. Bochenska M, Zielinska A, Kravtsov V C, Gdaniec M, Luks E and Radecka-Paryzek W 2002 Polyhedron 21763
6. Halouani H I, Dumazet-Bonnamour, Duchamp C, Bavoux C, Ehlinger N, Perrin M and Lamartine R 2002 Eur. J. Org. Chem. 4202

7. Casnati A, Baldini L, Pelizzi N, Rissanen K, Ugozzoli F and Ungaro R $2000 \mathrm{~J}$. Chem. Soc., Dalton Trans. 3411

8. Casnati A, Barboso S, Rouquette H, Schwing-Weill M J, Arnaud-Neu F and Dozol J F $2001 \mathrm{~J}$. Am. Chem. Soc. 12312182

9. Muzet N, Wipff G, Casnati A, Domiano L, Ungaro R and Ugozzoli F 1996 J. Chem. Soc. Perkin Trans. 2 1065

10. Beer P D, Drew M G B, Kan M, Leeson P B, Ogden $\mathrm{M} I$ and Williams G 1996 Inorg. Chem. 352202

11. Beer P D, Drew M G B and Ogden M I $1997 \mathrm{~J}$. Chem. Soc., Dalton Trans. 1489

12. Beer P D, Drew M G B, Leeson P B and Ogden M I 1996 Inorg. Chim. Acta 246133

13. Beer P D, Drew M G B, Grieve A and Ogden M I 1995 J. Chem. Soc., Dalton Trans. 3455

14. Arnaud-Neu F, Barrett G, Corry D, Cremin S, Ferguson G, Gallagher J F, Harris S J, McKervey M A and Schwing-Weill M J 1997 J. Chem. Soc. Perkin Trans. 2575

15. Beer P D, Drew M G B, Leeson P B and Ogden M I 1995 J. Chem. Soc., Dalton Trans. 1273

16. Ogden M I, Skelton B W and White A H $2001 \mathrm{~J}$. Chem. Soc., Dalton Trans. 3073

17. Collins E M, McKervey M A, Madigan E, Moran M B, Owens M, Ferguson G and Harris S J $1991 \mathrm{~J}$. Chem. Soc., Perkin Trans. 13137

18. Rao P V, Rao C P, Kolehmainen E, Wegelius E K and Rissanen K 2001 Chem. Lett. 111176

19. Gutsche C D and Iqbal M 1989 Org. Syn. 68 234

20. Casnati A, Fisher C, Guardigli M, Isernia A, Manet I, Sabbatini N and Ungaro R 1995 J. Chem. Soc., Perkin Trans. 2395

21. Ali A and Rao C P 2005 Indian J. Chem. B44 549

22. Arduini A, Ghidini E. Pochini A, Ungaro R, Andreetti G D, Calestani G and Ugozzoli F $1988 \mathrm{~J}$. Incl. Phenom. 6119

23. Hanna T A, Liu L, Zakharov L N, Rheingold A L, Watson W H and Gutsche C D 2002 Terahedron 58 9751

24. Andreetti A D, Pochini A and Ungaro R $1983 \mathrm{~J}$. Chem. Soc., Perkin Trans. 21773 